\title{
On the Algorithm for Equal Balls Packing into a Multi-connected Set
}

\author{
Alexander L. Kazakov \\ Matrosov Institute for \\ System Dynamics and \\ Control Theory of Siberian \\ Branch of the Russian \\ Academy of Sciences \\ Irkutsk, Russia \\ kazakov@icc.ru
}

Abstract - The paper is devoted to the problem of densest packing a given number of equal balls into multi-connected containers. The objective is to find the maximum radius associated with balls. We consider the problem both in three-dimensional Euclidean and one class of non-Euclidean spaces. In this study, the distance between points means the minimum time required to overcome the path between them. The algorithm based on the optical-geometric approach and billiard simulation combination is suggested and implemented. The idea is the following: we initially construct sufficiently small balls and enlarge them step by step as long as all the balls keep inside without overlaps. Computational experiments show the applicability and validity of the method.

Keywords - densest packing of balls, opticalgeometric approach, billiard simulation, threedimensional space, non-Euclidean space

\section{INTRODUCTION}

The problem of finding optimal packings is one of the classical problems of both optimization theory and computational geometry. The purpose is to arrange a finite number of given elements (often circles or balls) in a closed container without overlapping [1].

The problem of packing circles or spheres arises in various real-world applications [2]. The most obvious application is the packing bottles or cans into the smallest box. In materials science, random sphere packing is a model to represent the structure of liquids, proteins, and glassy materials. The model is used to study phenomena such as electrical conductivity, fluid flow, stress distribution and other mechanical properties of granular media, living cells, random media chemistry and physics [3]. In the car industry engineers want to minimize the size of a hole in the car body for a bundle of wires. The hole should be large enough for the bundle of wires to pass but not to be unnecessarily large because holes will weaken the body [4]. In the same way, it is of interest when it is necessary to pass bundles of cables or pipes through cylindrical shapes for very long distances. Since the smaller the diameter of the cylinders, the cheaper the cost.

\author{
Trung Thanh Ta \\ Irkutsk National Research \\ Technical University \\ Irkutsk, Russia \\ tatrungthanh@mail.ru
}

In logistics similar statements appear for a facility location problem or a segmentation of logistics service areas $[5,6]$. In city management it can be planting trees in a given region as to maximize the forest density [7].

There are two the most popular formulations for circle packing problem. The first one is to maximize the radius of the circles if the size and shape of the container are fixed. In the second case, it is necessary to minimize container size, if the radius of the circles is prescribed.

Lopez and Beasley [8] deal with the first statement and present a heuristic algorithm based on the formulation space search method to solve the packing problem for equal circles. The problem is formulated as a nonlinear optimization problem involving both Cartesian and polar coordinate systems.

As for the second formulation, we note papers [912]. Article [9] presents twice differentiable models for both two- and three-dimensional packing problems and proposes a method of their solving with the aid of ALGENCAN solver. In [10] by means of the resolution of non-linear equations systems through the Newton-Raphson method, the presented algorithm succeeds in improving the accuracy of previous results attained by continuous optimization approaches.

Al-Mudahka and Hifi [11] suggest an adaptive algorithm that incorporates nested partitioning within a Tabu search and applies some diversification strategies to obtain a (near) global optimum. The authors consider a circular container and present the results of a calculation with the number of circles up to 100 .

From the studies devoted to the three-dimensional packing problem, we note the following.

Gensane [13] proposes the billiard simulation approach to the packing problem of equal spheres inside a unit cube. Billiard simulation is a stochastic method that mimics the idealized movement of billiard balls inside a domain, with the initial centers of the balls and their directions being randomly fixed. To 
improve the convergence of the stochastic algorithm, different types of local searches are considered.

Papers $[14,15]$ consider the sphere packing problem as a nonlinear programming problem and approximately solve it using a hybrid heuristic which couples a variable neighborhood search with a local search. The approach provides accurate results with a numerical precision of $10^{-14}$

Tatarevic [16] deals with the problem of finding the densest packings of congruent, non-overlapping, spheres in a cube. The suggested method is based on a hypothesis that an improved packing can be reached just by the omission of two or more spheres from the cubic close-packed structure and by performing a shift of spheres using the available space made after we remove the spheres. The author presents several improved packings for $n \leq 4629$.

We note that most of the papers study the problem of packing in a simply connected space, and there are few publications deal with a multi-connected space.

Stoyan and Yaskov [17] deal with containers, which are circles with prohibited areas to be finite unions of circles of given radii. To find the local maxima, a modification of the Zoutendijk method of feasible directions and a strategy of active inequalities are applied. Because of prohibited areas, construction of starting points is essentially complicated. So a special way of constructing starting points is presented.

In [18] the authors consider the problem of packing a fixed number of identical circles inside the unit circle container with fixed size circular prohibited areas. The objective is to maximize the radius of the identical circles. The suggested algorithm is based upon formulation space search.

The vast majority of books and articles devoted to the study of the problem of packing in Euclidean space. In the case of non-Euclidean metrics this problem is weakly studied. Here we could mention the works [19] and [20], which deal with congruent circles packing problems for multidimensional spaces of a constant curvature and [21], which use the Hamming metric.

In this article, we propose an algorithm for solving the sphere packing problem for various bounded multi-connected containers in a three-dimensional non-Euclidean space. Here, as in our previous papers $[5,6,22,23]$, we use a special non-Euclidean metric, which means not the distance between points, but the time that is required to pass this way. Such statements appear in the logistics when one needs to locate a given number of commercial facilities and to maximize the overall service area.

\section{FORMULATION}

Let a bounded domain $D$ be defined in a threedimensional metric space $X$. There are non-empty sets $B_{j} \subset D, j=\overline{1, m}$. The closed set $P$ is defined as follows:

$$
P=\operatorname{cl}\left(D \backslash \bigcup_{j=1}^{m} B_{j}\right)
$$

Here $0 \leq f(x, y, z) \leq \beta$ is a piecewise continuous function, which specifies the instantaneous speed of movement at any point $T(x, y, z) \in D$. Sets $B_{j}, j=\overline{1, m}$ are called forbidden zones, and the speed of movement in these sets is zero: $f(x, y, z)=0 \Leftrightarrow T(x, y, z) \in B_{j}, j=\overline{1, m}$.

The distance between the points of the space $X$ is determined as follows:

$$
\rho(a, b)=\min _{G \in G(a, b)} \int_{G} \frac{d G}{f(x, y, z)},
$$

where $G(a, b)$ is the set of all continuous curves, which belong $X$ and connect the points $a$ and $b$.

Let $S_{i}, i=\overline{1, n}$, be congruent balls with centers $s_{i}\left(x_{i}, y_{i}, z_{i}\right)$ and radii $R$. It is necessary to find vector $s=\left(s_{1}, \ldots, s_{n}\right) \in \square^{3 n}$, which ensures the packing of the given number of balls with the maximum radius $R$ in $P$.

In other words, we have the following problem:

$$
\begin{array}{cc}
R \rightarrow \max & \\
\rho\left(s_{i}, s_{j}\right) \geq 2 R \quad \forall i=\overline{1, n}, \forall j=\overline{1, n}, i \neq j \\
\rho\left(s_{i}, \partial P\right) \geq R \quad \forall i=\overline{1, n} \\
s_{i} \in P & \forall i=\overline{1, n}
\end{array}
$$

Here $\partial P$ is the boundary of the set $P, \rho\left(s_{i}, \partial P\right)$ is the distance from a point to a closed surface.

\section{SOLUTION METHOD}

When one considers the packing problem in threedimensional space, it is important to be able to measure the distance from the point to the surface (as well as the distance between two points) with respect to the given metric.

From the point of view of geometrical optics, (1) means the time that is required for a light wave to pass the distance between points $a$ and $b$. According to the Huygens principle, any point of the $P$ reached by light can be regarded as an independent source of light. Therefore, by emitting a light wave from point $a$ one can construct its trajectory and fix the first photon reaching point $b$. By moving in the backward time, one can restore the trajectory of this photon which is the desired curve. A more detailed description of the algorithm can be found in [18] and for the threedimensional case, it is presented in [19].

In this paper, we propose an algorithm based on the principle of billiard modeling [20]. According to this principle, packing spheres are billiard balls that can move inside a given closed set. During the movement radius of the balls is increased. 


\section{A. Initialization}

Firstly, we randomly generate the initial coordinates of the centers of the balls $s_{i}\left(x_{i}, y_{i}, z_{i}\right), i=\overline{1, n}$ and calculate the distances between all pairs of points $\rho\left(s_{i}, s_{j}\right), i=\overline{1, n-1}, j=\overline{i+1, n}$ [18], as well as the distance $\rho\left(s_{i}, \partial P\right), i=\overline{1, n}$, from each point $s_{i}$ to the boundary of the set $P$ [19].

To ensure that the balls do not intersect each other, the initial radius value as follows:

$$
\begin{aligned}
& R_{0}=\min \left\{\rho\left(s_{k}, \partial P\right), \frac{\rho\left(s_{i}, s_{j}\right)}{2}\right\}, \\
& k=\overline{1, n}, i=\overline{1, n-1}, \quad j=\overline{i+1, n}
\end{aligned}
$$

Secondly, we construct balls $S_{i}, i=\overline{1, n}$, with radius $R_{0}$ and for each ball find the set of tangent points:

$$
\begin{gathered}
M_{i}=\left\{m \mid \rho\left(s_{i}, m\right)=R_{0}, m \in \partial P\right\} \cup\left\{m \mid m \in \partial S_{i}, m \in \partial S_{j}\right\}, \\
i=\overline{1, n}, j=\overline{1, n}, i \neq j .
\end{gathered}
$$

After initialization, one needs to move the balls to increase their radii.

\section{B. The algorithm of the shift of balls in a specified direction}

Suppose there are $n$ equal balls $S_{i}$ with centers $s_{i}, i=\overline{1, n}$ and radius $R$, which are found by the previous stage A.

It is easy to see that there is at least one ball, which has a tangency point, either with the boundary of the set $P$ or with another ball. If we push this ball away from the point of tangency, then it is possible to increase the radius. Using this idea, the centers of the balls move "as far as possible" from each other and from the boundary. This procedure ensures that the total radius of the balls does not decrease.

To determine the direction of the shift, one should follow the steps:

Step 1: Find a subset of balls whose set of tangent points is non-empty:

$$
S^{*}=\left\{S_{i} \mid M_{i} \neq \varnothing, i=\overline{1, n}\right\} .
$$

Step 2: For each ball of the subset $S^{*}$ we construct vectors whose origins are located in the center of the ball $s_{i}$, and the terminuses belong to the corresponding tangent points set $M_{i}$. If there are more than one such vectors, we find the sum vector $\overrightarrow{c_{i}}=\sum_{m \in M_{i}} \overrightarrow{s_{i} m}$.
Step 3: Move $s_{i}$ against the direction of $\overrightarrow{c_{i}}$ the distance $h$. The obtained point $s_{i}^{\prime}$ becomes the new center of the packed ball.

Step 4: Carry out the initialization stage with respect to the new centers $s_{i}^{\prime}, i=\overline{1, n}$.

Steps 1-4 are carried out as long as the radius increases. The algorithm is completed if the radius of the current iteration is less than the previous one. As a result we obtain the radius and coordinates of the centers of the packed balls.

\section{The algorithm of the shift of balls in a random direction}

As in the previous section, suppose there are $n$ equal balls $S_{i}$ with centers $s_{i}, i=\overline{1, n}$ and radius $R$.

According to a uniform grid constructing, there are 26 nearby points around each point (except points on the boundary). We assume that the ball center can be randomly moved to one of them.

The algorithm includes the following steps:

Step 1: Specify the maximum number of iterations, set the value of the iteration number counter to 1 .

Step 2: Find a subset of balls whose set of tangent points is non-empty $S^{*}$.

Step 3: For each center $s_{i}$ of $S_{i} \in S^{*}$ we randomly choose one of 26 nearby points. This point becomes a temporary center $\bar{s}_{i}$ of the corresponding ball.

Step 4: Find

$$
d^{i}=\min \left\{\rho\left(\bar{s}_{i}, \partial P\right), \frac{\rho\left(\bar{s}_{i}, s_{j}\right)}{2}\right\}, j=\overline{1, n}, i \neq j
$$

If $d^{i} \geq R$, we save the temporary center as the new center of the current ball and go to step 1 with the set $S^{*} / S_{j}$

If $d^{i}<R$, we return to step 3 and choose another point. If all 26 points have been considered, then we go to step 1 with the set $S^{*} / S_{j}$. In this case the center $s_{j}$ does not change.

Steps 3 and 4 are carried out for all balls from the set $S^{*}$.

Step 5: Recalculate the distances between all pairs of new points $\rho\left(\bar{s}_{i}, \bar{s}_{j}\right), i=\overline{1, n-1}, j=\overline{i+1, n}$, as well as the distance $\rho\left(\bar{s}_{i}, \partial P\right), i=\overline{1, n}$ and find a new radius $R^{\prime}$ by (2).

If $R^{\prime} \geq R$, the radius $R^{\prime}$ and the new coordinates $\bar{S}$ of the centers are saved as the current solution, and we go to step 2 .

If $R^{\prime}<R$, the value of the iteration counter increments. 
Step 6: If the value of the iteration counter becomes equal some preassigned value, then the algorithm is terminated. Otherwise, we go to step 2.

\section{The main algorithm}

Here we describe the general algorithm for packing $n$

spheres into a closed set. Note that achieving a global solution is not guaranteed. This feature inherited from the principles of billiard modeling.

Step 1: Approximate the surface function by the grid function using a uniform grid with a step $h$.

Step 2: Specify the maximum number of iterations, set the value of the iteration number counter Iter $=1$.

Step 3: Perform Initialization (stage $A$ ).

Step 4: Apply the algorithm of the shift of balls in a specified direction (stage $B$ ).

Step 5: Apply the algorithm of the shift of balls in a random direction (stage $C$ ).

Steps 4-5 are carried out as long as the radius increases.

Step 7: The counter of an initial solution generations Iter is incremented. If it becomes equal some preassigned value, then the algorithm is terminated. Otherwise, go to step 3.

As we mentioned above, a drawback of the algorithm is that it does not guarantee a solution globally maximizing the balls radii. We use multiple generating of initial positions to increase the probability of finding a global solution.

\section{COMPUTATIONAL EXPERIMENT}

In this section, we present some preliminary numerical results. The experiment is carried out on a personal computer with a Itel(R) Core(TM) i5-3570K $3.4 \mathrm{GHz}$ processor and 8GB RAM running on 64-bit Windows 7 operating system. CPU times are given in seconds. The algorithm is implemented in $\mathrm{C \#}$ programming language using Visual Studio 2012.

Example 1. To check the accuracy of the algorithm we pack equal balls into the unit sphere in the Euclidean metric space $(f(x, y, z) \equiv 1)$. Table I shows the comparison of our results $\left(R_{\max }\right)$ with the best known result $\left(R_{\text {known }}\right)$ from [21].

It should be noted that the calculation error depends on the grid step $h$. Here and further $h=0.001$. The deviation of the radius of the packed spheres is not greater than $1,15 \%$. The reduction of the grid step increases the computational accuracy, but calculation time increases significantly. In this example, with the number of balls $n=20$, the calculation time is $3 \mathrm{~h} 16 \mathrm{~min}$.
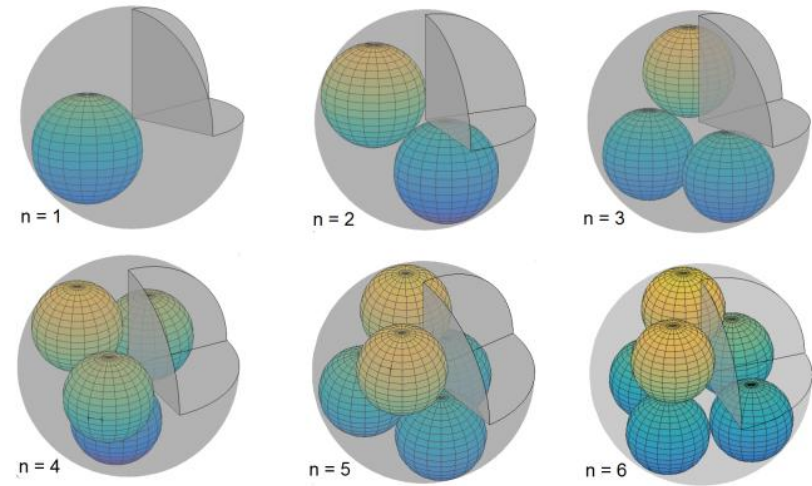

Fig. 1. Packing up to 6 equal balls into the unit sphere without the positive sector

TABLE I. COMPARISON OF PACKING RESULTS FOR THE UNIT SPHERE WITH THE EUCLIDEAN METRIC.

\begin{tabular}{|c|c|c|c|}
\hline $\mathbf{n}$ & $\mathbf{R}_{\mathbf{m a x}}$ & $\mathbf{R}_{\text {known }}$ & Relative error(\%) \\
\hline 2 & 0.2500 & 0.25000 & 0 \\
\hline 3 & 0.46394 & 0.46410 & 0.035 \\
\hline 4 & 0.44922 & 0.44948 & 0.060 \\
\hline 5 & 0.41354 & 0.41421 & 0.162 \\
\hline 6 & 0.41345 & 0.41421 & 0.184 \\
\hline 7 & 0.38439 & 0.38591 & 0.394 \\
\hline 8 & 0.37651 & 0.37802 & 0.400 \\
\hline 9 & 0.36455 & 0.36603 & 0.403 \\
\hline 10 & 0.35157 & 0.35305 & 0.419 \\
\hline 15 & 0.31482 & 0.31830 & 1.094 \\
\hline 20 & 0.28458 & 0.28789 & 1.149 \\
\hline
\end{tabular}

Example 2. Here we consider the problem of packing into a nonconvex set: a unit ball without a positive sector.

$$
P=\operatorname{cl}(D \backslash B),
$$

where

$$
D=\left\{(x, y, z): x^{2}+y^{2}+z^{2} \leq 1\right\}
$$
$B=\left\{(x, y, z) \in R^{3}: 0<x, y, z \leq 1\right\}$.

The results are shown in TABLE II. Fig. 1 illustrates the packings for $n$ balls, $n=\overline{1,6}$.

Considering Table II we can see that the highest density is achieved for $n=5$. As the number of balls increases, the packing density changes slightly.

Example 3. Here we deal with a unit cube with rectangular parallelepiped cutouts

$$
\begin{gathered}
P=\operatorname{cl}\left(D \backslash B_{1} \backslash B_{2}\right), \text { where } \\
D=\left\{(x, y, z) \in R^{3}: 0 \leq x, y, z \leq 1\right\}, \\
B_{1}=\left\{(x, y, z) \in R^{3}: 0 \leq x, y, z \leq 0.4\right\}, \\
B_{2}=\left\{(x, y, z) \in R^{3}: 0.8 \leq x, y, z \leq 1\right\} .
\end{gathered}
$$




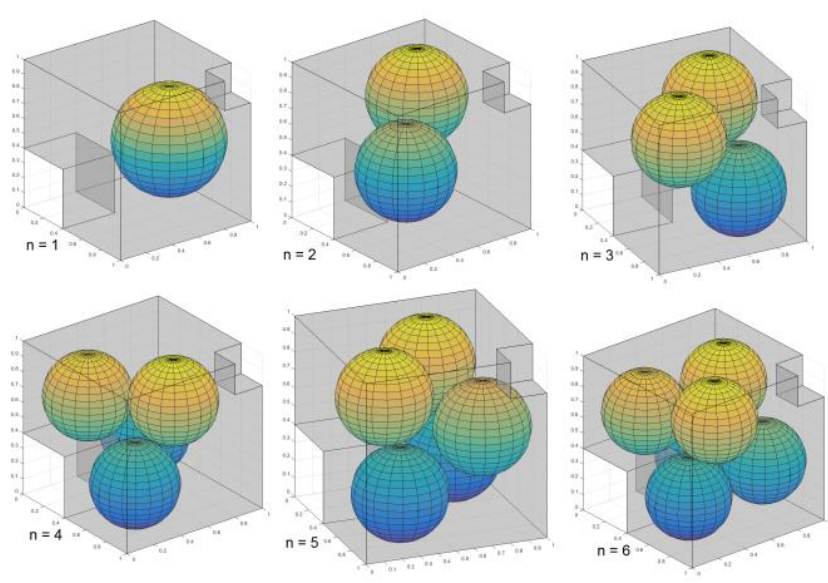

Fig. 2. Packing up to 6 equal balls into the unit cube with the cutouts.

TABLE II. PACKING EQUAL BALLS INSIDE THE UNIT SPHERE WITHOUT THE POSITIVE SECTOR.

\begin{tabular}{|c|c|c|c|}
\hline $\mathbf{n}$ & $\mathbf{R}_{\max }$ & Density $(\boldsymbol{\%})$ & $\mathbf{t}_{\text {executed }}$ \\
\hline 1 & 0.49998 & 16.6647 & 226 \\
\hline 2 & 0.47778 & 29.0839 & 464 \\
\hline 3 & 0.41526 & 28.6431 & 627 \\
\hline 4 & 0.41428 & 37.9210 & 1558 \\
\hline 5 & 0.41209 & 46.6536 & 1342 \\
\hline 6 & 0.37776 & 43.1259 & 1728 \\
\hline 7 & 0.35556 & 41.9542 & 2575 \\
\hline 8 & 0.35157 & 46.3508 & 2902 \\
\hline 9 & 0.33778 & 46.2502 & 3509 \\
\hline 10 & 0.32064 & 43.9533 & 4702 \\
\hline 15 & 0.27857 & 43.2338 & 6873 \\
\hline 20 & 0.24754 & 40.4487 & 9742 \\
\hline
\end{tabular}

The results are shown in TABLE II. Fig. 2 illustrates the packings for $n$ balls, $n=\overline{1,6}$.

The presence of non-convexities significantly complicates the task and does not allow constructing a package close to a cubic close-packed structure.

Example 4. Now we consider a multi-connected set. In this example, the container is a cube in which there are three forbidden zones of different types: a ball, a parallelepiped, and a nonconvex object bounded by a set of planes.

The results are shown in TABLE II. Fig. 3 The packing density increases with the number of balls, but the calculation time grows very quickly.

Example 5. Here we consider the sphere packing problem

for the non-Euclidean space. The metric is given by formula (1) where

$$
f(x, y, z)=x+y+1 .
$$
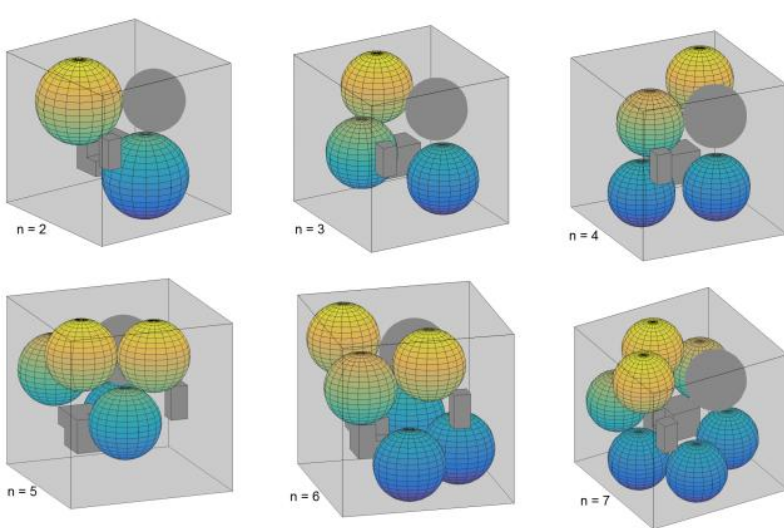

Fig. 3. Packing up to 6 equal balls into the multi-connected container.

TABLE III. PACKING EQUAL BALLS INSIDE THE UNIT UNIT CUBE WITH CUTOUTS

\begin{tabular}{|c|c|c|c|}
\hline $\mathbf{n}$ & $\mathbf{R}_{\max }$ & Density(\%) & $\mathbf{t}_{\text {executed }}$ \\
\hline 1 & 0.3600 & 21.0595 & 208 \\
\hline 2 & 0.3028 & 25.43762 & 469 \\
\hline 3 & 0.29289 & 34.02323 & 564 \\
\hline 4 & 0.26888 & 35.0975 & 855 \\
\hline 5 & 0.2567 & 38.17584 & 1224 \\
\hline 6 & 0.2554 & 45.11849 & 1712 \\
\hline 7 & 0.20946 & 29.05298 & 2242 \\
\hline 8 & 0.20148 & 29.53428 & 2544 \\
\hline 9 & 0.18354 & 25.11744 & 4705 \\
\hline 10 & 0.18247 & 27.42301 & 6457 \\
\hline
\end{tabular}

TABLE IV. PACKING EQUAL BALLS INSIDE THE MULTICONNECTED CONTAINER.

\begin{tabular}{|c|c|c|c|}
\hline $\mathbf{n}$ & $\mathbf{R}_{\max }$ & Density(\%) & $\mathbf{t}_{\text {executed }}$ \\
\hline 1 & 0.3000 & 11.7383 & 72 \\
\hline 2 & 0.2742 & 17.8864 & 517 \\
\hline 3 & 0.2330 & 15.8689 & 892 \\
\hline 4 & 0.2124 & 16.5695 & 1428 \\
\hline 5 & 0.2120 & 20.7119 & 2147 \\
\hline 6 & 0.2071 & 23.1368 & 3485 \\
\hline 7 & 0.2010 & 24.7131 & 4427 \\
\hline 8 & 0.1950 & 25.7891 & 6005 \\
\hline 9 & 0.1911 & 27.3065 & 8587 \\
\hline 10 & 0.1860 & 27.9757 & 9554 \\
\hline
\end{tabular}

It means that the light velocity linearly increases with respect to coordinates. In this metric space, the wave fronts have the shape of a sphere (as in Euclidean), but the center of the sphere shifts toward the origin. The container has the form 

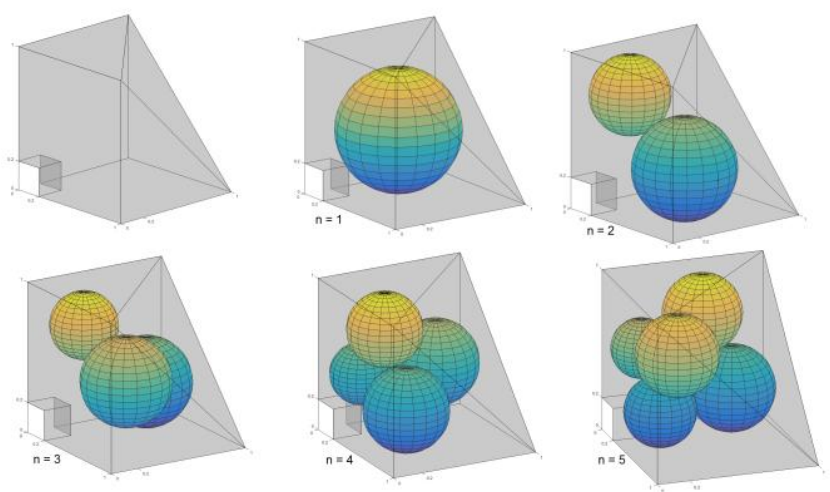

Fig. 4. Packing equal balls in the non-convex non-Euclidean container.

$$
P=\{(x, y, z): x+y+z \leq 2 ; 0 \leq x, y, z \leq 1\}
$$

with the prohibited area

$$
B=\left\{(x, y, z) \in R^{3}: 0 \leq x, y, z \leq 0.2\right\} .
$$

The results are shown in TABLE II. Fig. 4. Note that in Fig. 4 balls look different, but they have the same radius in a given metric.

TABLE V. PACKING EQUAL BALLS IN THE NON-CONVEX NONEUCLIDEAN CONTAINER..

\begin{tabular}{|c|c|c|c|}
\hline $\mathbf{n}$ & $\mathbf{R}_{\mathbf{m a x}}$ & Density(\%) & $\mathbf{t}_{\text {executed }}$ \\
\hline 1 & 0.2332 & 33.715 & 134 \\
\hline 2 & 0.1692 & 26.694 & 237 \\
\hline 3 & 0.1514 & 31.463 & 484 \\
\hline 4 & 0.1438 & 31.548 & 679 \\
\hline 5 & 0.1357 & 32.033 & 825 \\
\hline 6 & 0.1304 & 32.614 & 1537 \\
\hline 7 & 0.1227 & 32.011 & 2148 \\
\hline 8 & 0.1182 & 34.743 & 3325 \\
\hline 9 & 0.1089 & 32.881 & 5469 \\
\hline 10 & 0.1046 & 35.628 & 8254 \\
\hline
\end{tabular}

\section{CONCLUSIONS}

The sphere packing problem is a classic problem of mathematical optimization. In most publications, as a rule, the authors consider only simple containers, such as a cube, a sphere, a parallelepiped or a cylinder.

In this paper, we consider the problem of packing equal spheres into containers of various types, and the container may contain forbidden zones. The distance between points can be specified by the Euclidean metric, as well as non-Euclidean one. To solve this problem, we used an approach based on the physical principles of Fermat and Huygens, which allows us to determine the distance between points as the minimum time required to overcome the path between them. The proposed algorithm is based on a modified billiard simulation method.

The computational experiment shows that the algorithm is applicable both in the case of simple and complex multi-connected containers. The main advantage is the ability to use a special non-Euclidean metric, and the main drawback is the computation time. Our further research is aimed at overcoming this shortcoming.

\section{ACKNOWLEDGMENT}

The work is partially supported by RFBR, project No 18-07-00604.

\section{REFERENCES}

[1] J. H. Conway and N. J. A. Sloane, Sphere Packing, Lattice and Groups, 3rd ed., New York: Springer-Verlag, 1999.

[2] I. Castilo, F. Kampas and J. Pinter, "Solving circle packing problems by global optimization: Numerical results and industrial applications," in European Journal of Operational Research, vol. 191(3), 2008, pp. 786-802.

[3] M. Gan, N. Gopinathan, X. Jia, and R. A. Williams, "Predicting packing characteristics of particles of arbitrary shapes," in KONA Powder \& Particle Journal, vol. 22, 2004, pp. 82-93.

[4] K. Sugihara, M. Sawai, H. Sano, D.S. Kim, and D. Kim, "Disk packing for the estimation of the size of a wire bundle," in Japan Journal of Industrial and Applied Mathematics, vol. 21(3), 2004, pp. 259-278.

[5] A. L. Kazakov and A. A. Lempert, "On mathematical models for optimization problem of logistics infrastructure," in International Journal of Artificial Intelligence, vol.13(1), 2015, pp. 200-210.

[6] A. L. Kazakov, A. A. Lempert, and D. S. Bukharov, "On segmenting logistical zones for servicing continuously developed consumers," in Automation and Remote Control, vol. 74(6), 2013, pp. 968-977.

[7] P. Szabo, M. Markot, T. Csendes, E. Specht, and L. Casado, "New approaches to circle packing in square with program codes," in Springer US, New York.

[8] C. Lopez and J. Beasley, "A heuristic for the circle packing problem with a variety of containers," in European Journal of Operational Research, vol. 214(3), 2011, pp. 512-525.

[9] E. Birgin and F. Sobral, "Minimizing the object dimensions in circle and sphere packing problems," in Computers \& Operations Research, vol. 35(7), 2008, pp. 2357-2375.

[10] E. Birgin and J. Gentil, "New and improved results for packing identical unitary radius circles within triangles, rectangles and strips," in Computers \& Operations Research, vol. 37(7), 2010, pp. 1318-1327.

[11] I. Al-Modahka, M. Hifi, and R. M'Hallah, "Packing circles in the smallest circle: an adaptive hybrid algorithm," in Journal of the Operational Research Society, vol. 62(11), 2011, pp.1917-1930.

[12] J. J. Flores, J. Martínez, and F. Calderón, "Evolutionary computation solutions to the circle packing problem," in Soft Comput, vol. 24(4), 2016, pp. 1521-1535.

[13] T. Gensane, "Dense packings of equal spheres in a cube," in Electronic Journal of Combinatorics, vol. 11(1), 2004, R33.

[14] R. M'Hallah and A. Alkandari, "Packing unit spheres into a cube using VNS," in Electronic Notes in Discrete Mathematics, vol. 39C 2012, pp. 201-208.

[15] R. M'Hallah, A. Alkandari and N. Mladenovic, "Packing unit spheres into the smallest sphere using VNS and NLP," in Computers Operations Research, vol. 40, 2013, pp. 603-615.

[16] M. Tatarevic, “On Limits of Dense Packing of Equal Spheres in a Cube," in The Electronic Journal of Combinatorics, vol. 22(1), 2015, pp. 35

[17] Y. Stoyan and G. Yaskov, "Packing equal circles into a circle with circular prohibited areas," in International Journal of Computer Mathematics, vol. 89(10), 2012, pp. 1355-1369.

[18] C. Lopez and J. E. Beasley, "Packing a fixed number of identical circles in a circular container with circular prohibited areas," in arXiv e-prints, 2018, arXiv:1811.00622. 
[19] H. S. M. Coxeter, "Arrangements of equal spheres in noneuclidean spaces," in Acta Mathematica Academiae Scientiarum Hungarica, vol. 5(3), 1954, pp. 263-274.

[20] K. Boroczky, "Packing of spheres in spaces of constant curvature," in Acta Mathematica Academiae Scientiarum Hungarica, vol. 32(3), 1978, pp.243-261.

[21] R. McEliece and H. Rumsey, "Sphere-packing in the hamming metric," in Bull. American Math. Soc., vol. 75, 1969, pp. 32-34.

[22] A. L. Kazakov and A. A. Lempert, "An approach to optimization in transport logistics," in Automation and Remote Control, vol. 72(7) 2011, pp. 50-57.

[23] A. L. Kazakov, A. A. Lempert and T. T. Ta, "The sphere packing problem into bounded containers in three-dimension non-Euclidean space," in IFAC-PapersOnLine, vol. 51(32), 2018, pp. 782-787.

[24] R. L. Graham, B. D. Lubachevsky, K. J. Nurmela and P. R. J Ostergard, "Dense packings of congruent circles in a circle," in Discrete Mathematics, vol. 181, 1998, pp. 139-154.

[25] H. Pfoertner, "Densest packings of $n$ equal spheres in a sphere of radius 1,” in www.randomwalk.de/sphere/ insphr/spisbest.txt, 2008, accessed 2 February 2019. 